ISSN: 2711--1792 (En línea) • Espacio Matemático Vol. 1 No. 2 (2020), pp. 92-99.

\title{
Assessment and Methods for Solving the Problems of the Republican Mathematical Olympiad for Schoolchildren in Kyrgyzstan
}

\author{
Aida O. Keldibekova, Ulanbek A. Sopuev
}

\begin{abstract}
The article studies methods for solving, criteria for assessment of the real issues of the 2019 Republican Mathematics Olympiad in the Kyrgyzstan using a quantitative scale with a given number of points. As a result of the Olympiad, 318 winners were determined in 10 school subjects, which makes up $15.58 \%$ of the participants in the regional stage. 73 students won the individual classification, that is $3.57 \%$ of all participants, starting from the regional stage. Seven winners were recognized in mathematics.
\end{abstract}

Key words and phrases: olympiad, mathematics, problem, regional stage, final stage, solution, evaluation criteria.

\section{Evaluación y Métodos para Resolver los Problemas de la Olimpiada Republicana de Matemática para escolares en Kyrgyzstan}

\section{Resumen}

Este artículo estudia métodos de resolución y criterios de evaluación para los temas de la Olimpiada Republicana de Matemática 2019 en Kyrgyzstan, usando una escala cuantitativa con un número dado de puntos. Como resultado de la Olimpiada, se determinaron 318 ganadores en 10 temas escolares, lo que hace el $15.58 \%$ de los participantes en la fase regional. 73 estudiantes alcanzaron la clasificación individual, esto es el 3.57\% del total de participantes, a partir de la fase regional. Siete ganadores lo fueron en matemática.

Palabras y frases clave: Olimpiada, matemática, problema, fase regional, fase final, solución, criterios de evaluación.

\section{Introduction}

In connection with the increasing complexity of the Olympiad tasks in recent years, there is a need to introduce schoolchildren to new methods for solving tasks of the Olympiad character in the system of additional education. At the same time realization of student's 
need for success will allow to design work on the orientation of the educational process on the achievements of schoolchildren. In the research the following conclusions were drawn: the content of the competition tasks corresponds to the composition and logical structure of mathematical competence, the competition problem acts as a tool that determines the level of subject and key competencies formation (Keldibekova, 2017).Criteria assessment of tasks of mathematical Olympiads is based on expert assessment, reflecting levels of knowledge of mathematical competencies (Keldibekova, 2019). Criteria assessment of the Olympiad works should correspond to the form of detailed control, revealing the student's ability to solve complex problems, their individual psychological and pedagogical features and mathematical abilities. The concreteness and accuracy of the wording of the criteria for assessing tasks, determining points for each stage of solving the problems of the Olympiad contributes to an objective assessment of the mathematical abilities of its participants, providing a better selection of the winners of the final stage.

In the practice of criteria-based assessment, three approaches to assessing the solution of the Olympiad problem have been identified, which are based on the level of difficulty of the tasks. So, in the republican Olympiads of Kyrgyzstan and Russia in mathematics, 7-, 10-point systems for assessing problem solving are traditionally used(Farkov, 2013, p. 16-18, 83-84), (Keldibekova, 2019), there is an estimate of the complexity of the problem according to a 30-point system (Gorbachev, 2004).The article authorsshare the methodical experience of working in the jury of city and regional schoolchildren competitions, and determines the criteria for an objective evaluation of schoolchildren competition works, which are used by the competition commissions grading each task.

The purpose of the research is to demonstrate solution methods and criteria for assessing the Olympiad problems of the regional and final stages of the republican Olympiad of schoolchildren in mathematics in the Kyrgyz Republic, held in 2019.

\section{Solution methods and evaluation criteria for solving problems of the regional stage of the mathematical competition.}

In this section, we show examples of solving some problems of the regional stage of the 2019 Olympiad with a description of the assessment criteria for each problem.

Problem 1. Which of the numbers is greater: $\left(2019+20^{19}\right)$ or $19^{20}$ ?

Solution to problem 1. The general idea of the solution: the value of the number $20^{19}$ is significantly less than the value of the number $19^{20}$, so adding the number 2019 does not change the ratio. Two methods of proof are possible.

Method 1. Estimate the value of the number using Newton's bin:

$$
\begin{aligned}
20^{19}=(19+ & 1)^{19} \\
& =19^{19}+\left\{19 \cdot 19^{18}+\left(19 \cdot \frac{18}{2}\right) \cdot 19^{17}+\cdots+\left(19 \cdot 18 \cdot \ldots \cdot \frac{3}{17 !}\right) \times 19^{2}\right\} \\
& + \\
+19 \cdot 19+1 & <19^{19}+\left\{19 \cdot 19^{18}+(19 \cdot 18) \cdot 19^{17}+\cdots+(19 \cdot 18 \cdot \ldots \cdot 3) \times 19^{2}\right\} \\
& +361+1 \\
& \Rightarrow 20^{19}<19^{19}+17 \cdot 19^{19}+361+1=18 \cdot 19^{19}+362
\end{aligned}
$$

In curly brackets we have 17 terms, each of which is less than $20^{19}$ 
$2019+20^{19}<18 \cdot 19^{19}+362+2019=18 \cdot 19^{19}+2381<18 \cdot 19^{19}+19^{19}=19^{20}$

Method 2.Let's divide both numbers by $20^{19}$. Let's estimate the value of the number:

$$
\begin{aligned}
& \frac{2019}{19^{19}}+\left(1+\frac{1}{19}\right)^{19}<1+\left(\left(1+\frac{1}{19}\right)^{2}\right)^{9}\left(1+\frac{1}{19}\right)<1+\left(1+\frac{1}{9}\right)^{9}\left(1+\frac{1}{19}\right)<1+ \\
+ & \left(1+\frac{1}{9}\right)^{10}<1+\left(\left(1+\frac{1}{9}\right)^{2}\right)^{5}<1+\left(1+\frac{1}{4}\right)^{5}<1+1,6^{2} \cdot 1,6<1+3 \cdot 1,6<7<19
\end{aligned}
$$

Thus, we have proved that $2019+20^{19}<19^{20}$.

Answer: the second number is greater.

Evaluation criteria for problem 1: when checking, you need to pay attention to ensure that all calculations are not with rounding numbers, but with their evaluation in the right direction.

For the correct answer without calculation and proof, 1 point is awarded.

For a correct answer with incomplete proof, but with correct calculations, up to 5 points are awarded at the discretion of the jury.

For full proof, 10 points are awarded.

Problem 2.a) How many five-digit numbers exist, the digits of which can only be 2, 1 or 0 (for example, 20000, 12222, 20100)?

b) Find the sum of these numbers.

\section{Solution to problem 2}

a) Method 1. These five-digit numbers have 1 or 2 on the left position. Each of the remaining four positions has any of the three valid digits. Therefore, the total number of these five-digit numbers is:

$$
X=2 \cdot 3 \cdot 3 \cdot 3 \cdot 3=162
$$

Method 2. We apply the ternary number system. The smallest allowed number is $1000_{3}=$ $1 \cdot 3^{4}=81$.The largest number allowed

$$
22222_{3}=2 \cdot 3^{4}+2 \cdot 3^{3}+2 \cdot 3^{2}+2 \cdot 3^{1}+2=162+54+18+6+2=242 .
$$

The total number of $242-81+1=162$ (please note, in this place it is possible to make a mistake of 1).

Answer: 162.

b) At the position of units of these allowable digits equally, 54 each. Their sum is equal $S_{1}=2 \cdot 54+1 \cdot 54+0 \cdot 54=162$.

The sums $S_{2}, S_{3}, S_{4}$ at the positions of tens, hundreds and thousands will be respectively $S_{2}=1620, S_{3}=16200, S_{4}=162000$. At the position of tens of thousands of digits 2 and 1 equally, 81 each. Their sum is equal $S_{5}=2 \cdot 81 \cdot 10000+1 \cdot 81 \cdot 10000=$ 2430000. The sum of all these five-digit numbers is: $S=S_{1}+S_{2}+S_{3}+S_{4}+S_{5}=$ 2609982.

Evaluation criteria for problem 2.

Answer: 2609982.

a) for the correct answer 162, without justification, 1 point is awarded; with justification for the answer - 5 points. 
b) for the correct answer without justification 1 point; with justification - 5 points.

For the entire task, with informed decisions of parts a and $b-10$ points.

\section{Methods of solution and evaluation criteria for solving problems of the republican stage of the mathematical completion}

Let's consider the criteria for evaluating solutions to problems on a 10-point system using real tasks as the final tasks of the final stage of the mathematics Olympiad.

Problem 3. By what formula can the function $f(x)$ be calculated, if its values are known for some values of the argument:

$$
f(-1)=1, f(0)=3, f(1)=1, f(2)=3 \text {. }
$$

Find one or two different formulas.

\section{Solution to problem 3.}

Method 1. We represent the function $f(x)$ in the form of a polynomial of degree 3:

$$
f(x)=a x^{3}+b x^{2}+c x+d \text {. }
$$

Giving the argument $x$ values $-1 ; 0 ; 1 ; 2$, we get the system:

$$
\left\{\begin{array} { l } 
{ a ( - 1 ) ^ { 3 } + b ( - 1 ) ^ { 2 } + c ( - 1 ) + d = 1 ; } \\
{ a ( 0 ) ^ { 3 } + b ( 0 ) ^ { 2 } + c ( 0 ) + d = 3 ; } \\
{ a ( 1 ) ^ { 3 } + b ( 1 ) ^ { 2 } + c ( 1 ) + d = 1 ; } \\
{ a ( 2 ) ^ { 3 } + b ( 2 ) ^ { 2 } + c ( 2 ) + d = 3 . }
\end{array} \Rightarrow \left\{\begin{array} { l } 
{ - a + b - c + d = 1 ; } \\
{ d = 3 ; } \\
{ a + b + c + d = 1 ; } \\
{ 8 a + 4 b + 2 c + d = 3 . }
\end{array} \Rightarrow \left\{\begin{array}{l}
a=\frac{4}{3} ; \\
b=-2 ; \\
c=-\frac{4}{3} ; \\
d=3 .
\end{array}\right.\right.\right.
$$

Then $f(x)=\frac{4}{3} x^{3}-2 x^{2}-\frac{4}{3} x+3$.

Method 2. One can represent $f(x)$ as a periodic function, for example:

$f(x)=a \cos b x+c$. The possible answer is $f(x)=\cos \pi x+2$.

Method 3. You can use periodicity using the function "fractional part of a number". In this case, the jury must take into account that this function does not have a standard designation, and we must agree with the designation proposed by the participant.

Method 4. You can imagine the function $f(x)$ in other forms, for example:

$$
f(x)=a|x|+b|x-1|+c x+d
$$

Answer: 1) $f(x)=\frac{4}{3} x^{3}-2 x^{2}-\frac{4}{3} x+3$.

\section{Criteria for assessing problem 3}

A notation of the form $f(x)=\cdots(x<\cdots) \ldots(x>\cdots)$ is not a formula. For such an answer 1 point is awarded.

- For one solution 5 points are awarded.

- For two similar options 6 points are awarded.

- For two different options 10 points are awarded.

Problem 4. a) Find all possible unequal tetrahedrons with five edges, lengths of which are $19,19,10,10,10$, and the sixth edge has a length of 19 or 10. 
b) Find the volume of one of the resulting tetrahedrons and round it to an integer with an excess.

\section{Solution to problem 4}

a) 1.1. If there are two ribs of 19 each and four ribs of 10 each, then the ribs of 19 each may have a common vertex. Such a tetrahedron exists.

Answer 1.1. Two ribs of 19 with a common vertex, the remaining ribs of 10 .

1.2. If the ribs of 19 do not have a common vertex, then all four triangles are the same and have sides 19,10,10. Such a tetrahedron does not exist, since two small angles smaller than $45^{\circ}$, converge at the vertex and an obtuse angle.

2. In the tetrahedron, ribs 19, 19, 19, 10, 10, 10.

2.1. If three edges of 10 each come from one vertex, then three others of 19 each form the base of the pyramid - a regular triangle of large area. Three other triangles with sides 10 , 10,19 in total have a smaller area than the base area; such a pyramid does not exist.

2.2. If there are no identical edges emerging from one vertex, then in the tetrahedron $A B C D$ the edges of the same length can be located along broken lines, for example, $A B=B C=C D=10, C A=A D=D B=16$. Such a tetrahedron does not exist.

2.3. The last case is a regular pyramid with side ribs equal to 19 and base ribs equal to 10 . b) $V=\frac{25}{3} \cdot \sqrt{983} \approx 262$ - the volume of the pyramid described in paragraph 2.3 .

Answer: a) There are 2 tetrahedra of this type, cases 1.1 . and 2.3 ; b) $V \approx 262$.

\section{Criteria for assessing problem 4}

a) For establishing the existence of each of these two tetrahedra with their exact description, up to 3 points are awarded, i.e. 6 points;

- for the correct proof that other options do not exist, accrued up to 2 points,

b) up to 2 points are awarded for calculating the volume of an existing tetrahedron.

\section{Results of the regional and final stages of the 2019 republican Olympiad}

The organization of the republican Olympiad for schoolchildren of the Kyrgyz Republic goes through 4 stages (Keldibekova \& Baisalov, 2019). The republican Olympiad for schoolchildren of the 2018-2019 schooling year started in October 2018.

The regional stage of the 2019 republican school Olympiad was held in two rounds on March 12-13 in all regions of Kyrgyzstan. In the regional stage of the Olympiad, 2041 schoolchildren of the republic participated, of which 171 were students of secondary schools, gymnasiums, lyceums in Osh, 177 students came from schools of the city of Bishkek, and 1693 school's students from the regions of the republic.

The final stage of the republican Olympiad for schoolchildren of the Kyrgyz Republic was held in two rounds on March 30-31, 2019. Competing in 10 school subjects, 318 winners of the regional stage, held in Bishkek, Osh, and the country's regions (i.e. $15.58 \%$ of the total number of participants in the regional stage) took part in it. According to the results of the final stage, 9 regions of the republic showed the following results, fig. 2:

\section{Figure 2}




\section{Results of the 2019 republican Olympiad by region}

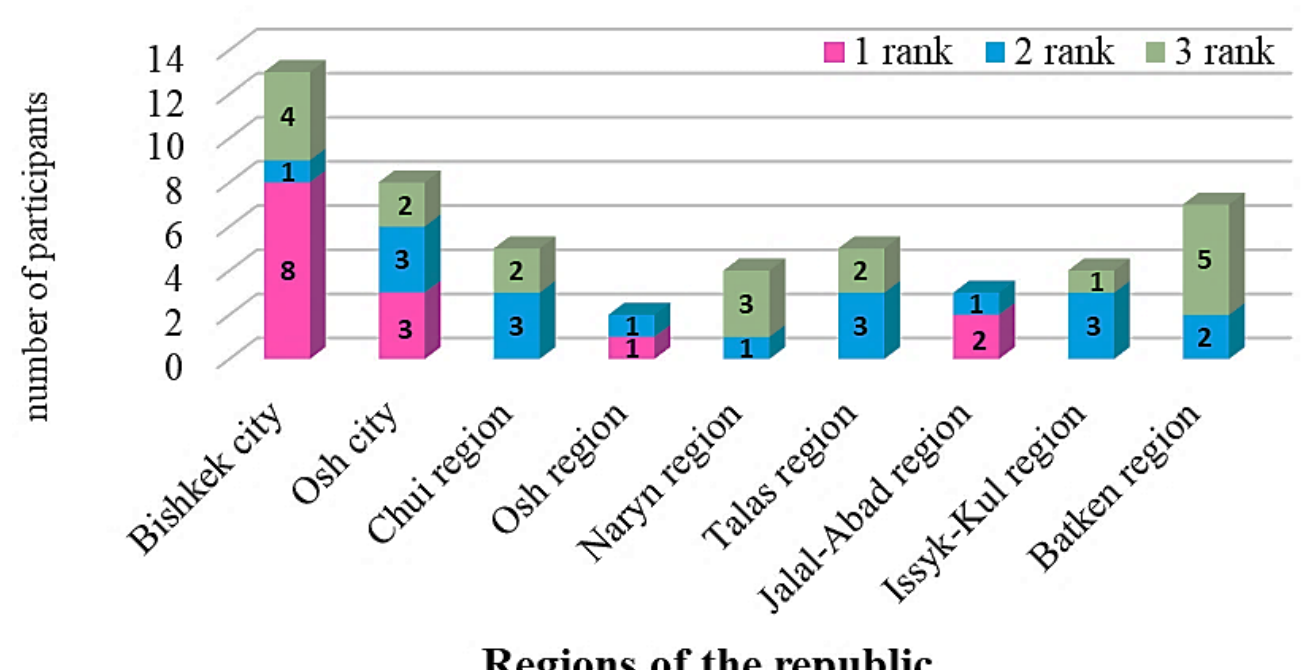

The team championship belongs to Bishkek. The second place was shared by the teams of Olympians of Osh and Issyk-Kul regions; the third place was taken by students of the Batken region, fig. 3:

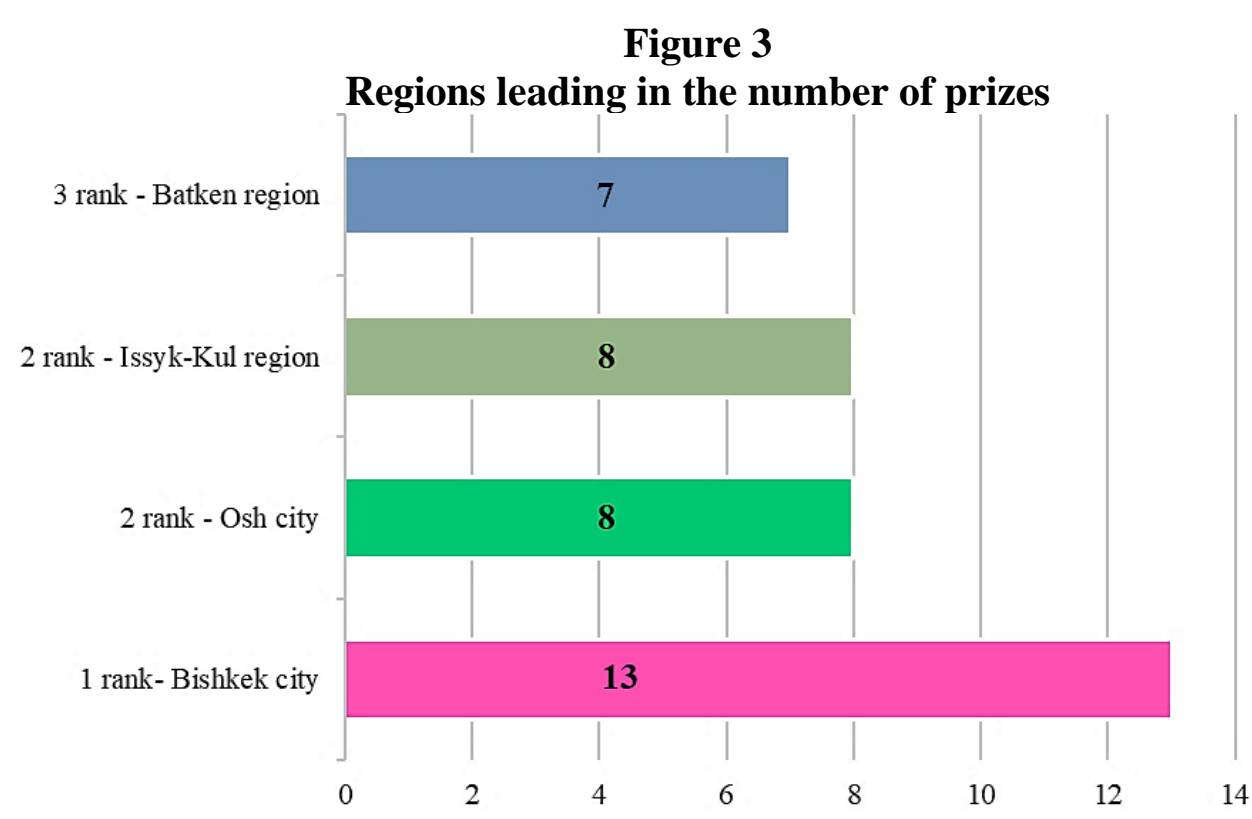

73 students became winners of the final stage of the Olympiad in the individual classification, which is $3.57 \%$ of the total number of participants, starting from the regional stage of the Olympiad, fig. 4. 
ISSN: 2711--1792 (En línea) • Espacio Matemático Vol. 1 No. 2 (2020), pp. 92-99.

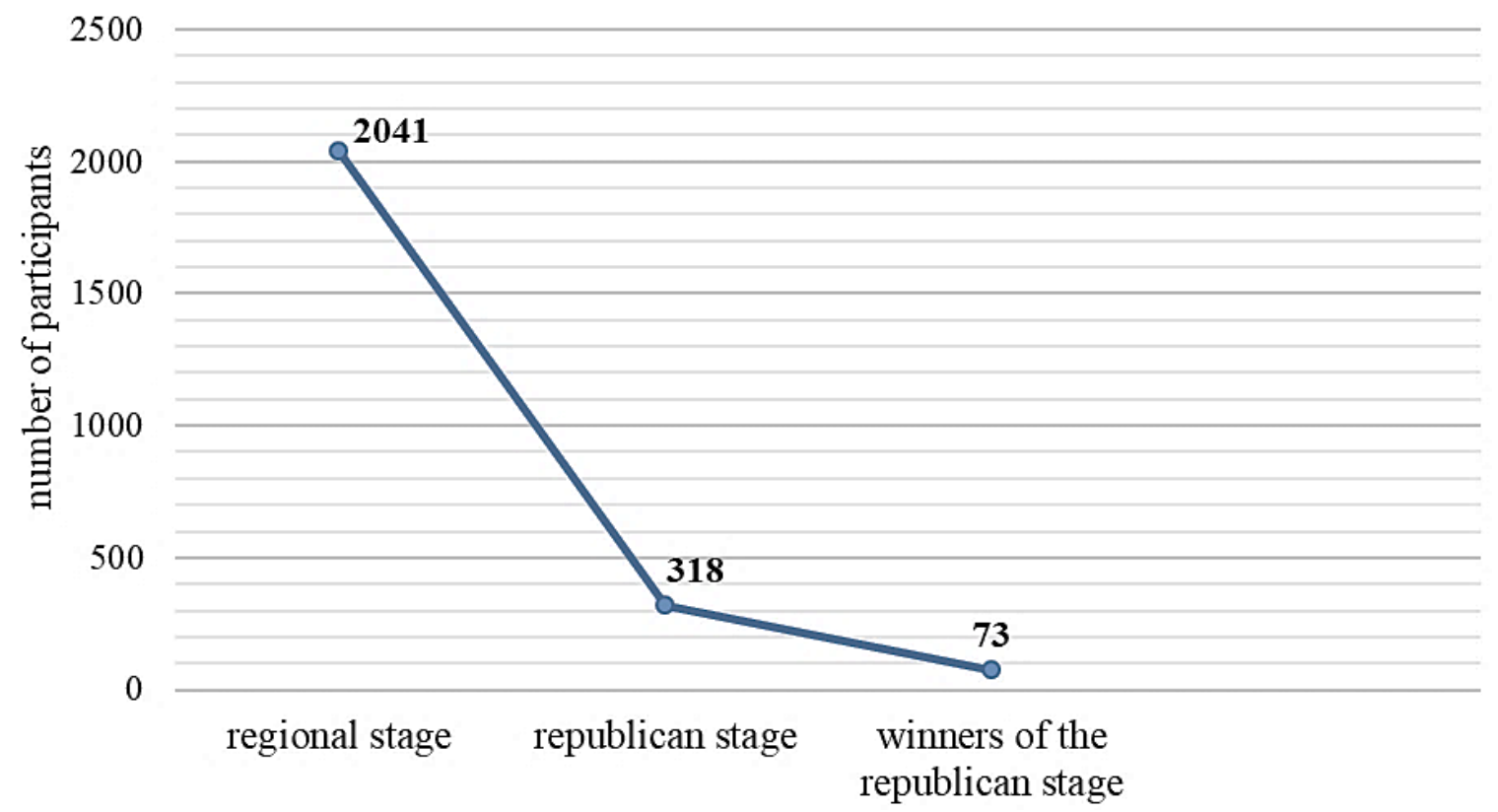

Compared with the Olympiads of previous years, there was an increase in the number of winners from the regions of Kyrgyzstan. Fig. 5 shows the distribution of prizes in the subject of mathematics:

Figure 5

\section{Results of the 2019 math Olympiad}

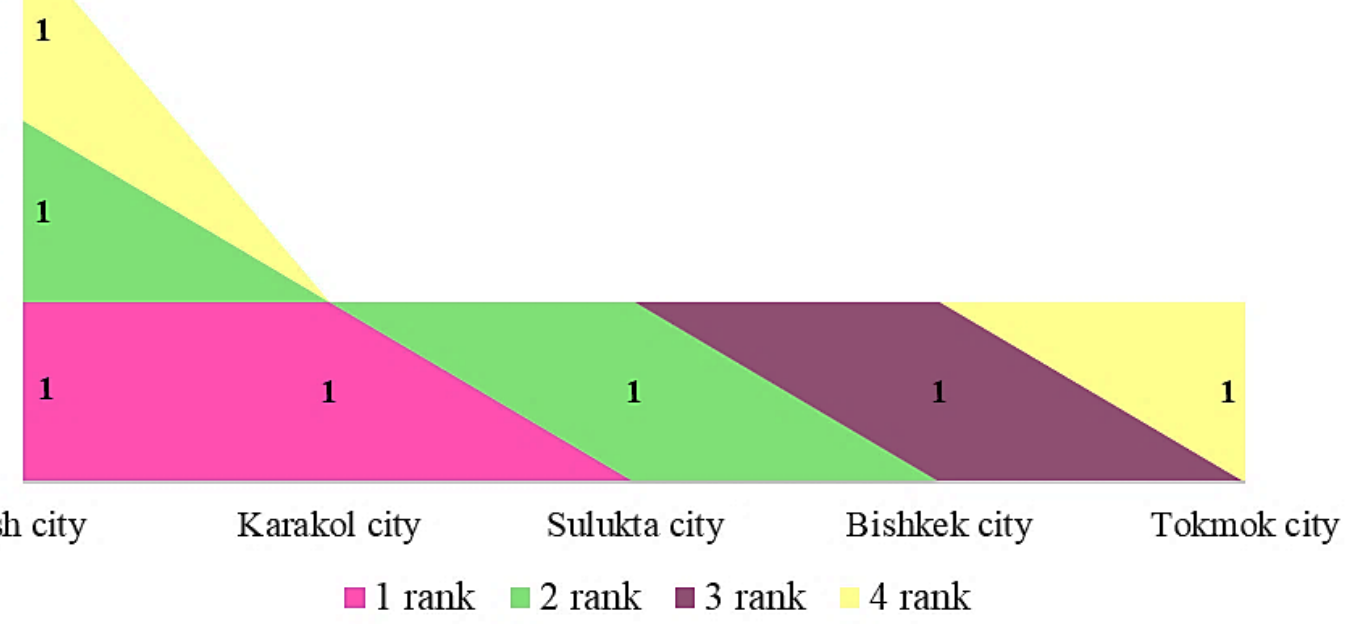

In the subject of mathematics 7 students won prizes: 2 students $-1^{\text {st }}$ place, 2 students $-2^{\text {nd }}$ place, 1 student $-3^{\text {rd }}$ place, 2 students $-4^{\text {th }}$ place. First place in the team standings, took the students of the city of Osh.

\section{Conclusions}

An objective assessment of the Olympiad work is ensured by the conformity of the evaluating factors, the features by which an unambiguous assessment is made, and the use of adequate meters. Assessment of written / practical / oral assignments and appeals of the 
ISSN: 2711--1792 (En línea) • Espacio Matemático Vol. 1 No. 2 (2020), pp. 92-99.

2019 of the Kyrgyz Republican Mathematical Olympiad was based on criteria developed by the Center for Assessment in Education and teaching methods.

The members of the jury need methodical instructions in determining the criteria for evaluating schoolchildren competition activity and for methods of solutions and types of mistakes.

Acknowledgements. We bring our sincere appreciation to the reviewers of this article for comments and recommendations, as well as providing editorial assistance.

\section{References}

1. Keldibekova,A.O. \& Baysalov, J. U, Republican Olympiad of schoolchildren in Kyrgyzstan: principles, features, innovations and results, Modern high technologies (4) (2019), 118-128. DOI: https://doi.org/10.17513/snt.37503

2. Farkov, A.V., Mathematical Olympiads. 5-6 grades. Moscow 2013, Exam Publ.

3. Keldibekova, A.O. On approaches to assessing the solution of problems in mathematical Olympiads for schoolchildren. Perspectives of Science and Education. 41(5) (2019), 324-344. DOI: https://doi.org/10.32744/pse.2019.5.23

4. Gorbachev, N.V., Collection of Olympiad problems in mathematics. Moscow 2004, ICMMO Publ.

Aida O. Keldibekova (aidaoskk@gmail.com)

Osh Stateuniversity,Kyrgyzstan.

Ulanbek A. Sopuev (ulansopuev@mail.ru)

Osh Stateuniversity, Kyrgyzstan. 